\title{
Aditivos químicos em alimentos ultraprocessados e os riscos à saúde infantil
}

\author{
Chemical additives in ultraprocessed foods and the risks to child health
}

Aditivos químicos en alimentos ultraprocesados y los riesgos para la salud infantil

Natiele Bezerra Silva ${ }^{1}$, Valéria Magna das Chagas Moura', Daniela Fortes Neves Ibiapina ${ }^{1}$, Keila Cristiane Batista Bezerra'.

\section{RESUMO}

Objetivo: Analisar rótulos de alimentos ultraprocessados voltados à população infantil quanto à presença de aditivos químicos e identificar na literatura científica os riscos que podem oferecer a saúde infantil. Método: Trata-se de um estudo de delineamento transversal, de caráter descritivo qualitativo e quantitativo, onde foram analisados 51 rótulos de produtos utraprocessados. Os dados foram coletados através da busca dos rótulos nos endereços eletrônicos das marcas selecionadas de cada alimento. Resultados: As três classes de aditivos mais encontradas foram os aromatizantes, encontrado em maior quantidade, estando descrito na rotulagem de 50 produtos (98\%), seguido dos corantes encontrados em 34 produtos (67\%) e dos acidulantes, presentes em 28 produtos (55\%). Observou-se que consumo em excesso desses aditivos pode ocasionar problemas de saúde nas crianças como alergias, com a presença de urticária, angioedema, broncoespasmo e choque, Transtorno do Déficit de Atenção e Hiperatividade, retardo do crescimento infantil, vários tipos de câncer, descalcificação dos dentes e dos ossos, que levam ao enfraquecimento, entre outros. Conclusão: As classes e tipos de aditivos encontrados em maior prevalência nos produtos alimentícios analisados estão relacionados a diversos prejuízos a saúde infantil.

Palavras-chave: Alimentação Infantil, Aditivos Alimentares, Saúde da Criança.

\begin{abstract}
Objective: To analyze labels of ultraprocessed food products aimed at the child population regarding the presence of chemical additives and to identify in the scientific literature the risks that children's health can offer. Method: This is a cross-sectional, qualitative and quantitative descriptive study, where 51 labels of utraprocessed products were analyzed. The data were collected through the search of the labels in the electronic addresses of the selected brands of each food. Results: The three classes of additives found were the most commonly found in the labeling of 50 products (98\%), followed by the dyes found in 34 products $(67 \%)$ and acidulants present in 28 products $(55 \%)$. It was observed that excessive consumption of these additives can cause health problems in children as allergies, with the presence of urticaria, angioedema, bronchospasm and shock, Attention Deficit Hyperactivity Disorder, infant growth retardation, various types of cancer, descaling of teeth and bones, leading to weakening, among others. Conclusion: The classes and types of additives found in higher prevalence in the analyzed food products are related to several damages to children's health.
\end{abstract}

Key words: Infant Feeding, Food Additives, Child Health.

${ }^{1}$ Centro Universitário Santo Agostinho, Teresina-PI. *E-mail: natielebezerra@gmail.com 


\section{RESUMEN}

Objetivo: Analizar rótulos de alimentos ultraprocesados dirigidos a la población infantil en cuanto a la presencia de aditivos químicos e identificar en la literatura científica los riesgos que pueden ofrecer la salud infantil. Método: Se trata de un estudio de delineamiento transversal, de carácter descriptivo cualitativo y cuantitativo, donde se analizaron 51 etiquetas de productos utraprocesados. Los datos fueron recolectados a través de la búsqueda de las etiquetas en las direcciones electrónicas de las marcas seleccionadas de cada alimento. Resultados: Las tres clases de aditivos más encontrados fueron los aromatizantes, encontrados en mayor cantidad, estando descrito en el etiquetado de 50 productos (98\%), seguido de los colorantes encontrados en 34 productos (67\%) y de los acidulantes, presentes en 28 productos $55 \%$ ). Se observó que el consumo excesivo de estos aditivos puede ocasionar problemas de salud en los niños como alergias, con la presencia de urticaria, angioedema, broncoespasmo y shock, trastorno del déficit de atención y hiperactividad, retraso del crecimiento infantil, varios tipos de cáncer, descalcificación de los dientes y de los huesos, que llevan al debilitamiento, entre otros. Conclusión: Las clases y tipos de aditivos encontrados en mayor prevalencia en los productos alimenticios analizados están relacionados a diversos daños a la salud infantil.

Palabras-clave: Alimentación Infantil, Aditivos alimentarios, Salud del Niño.

\section{INTRODUÇÃO}

A alimentação infantil é um tema que nos últimos anos tem despertado grande interesse em várias áreas do conhecimento por envolver diferentes aspectos além dos nutricionais. O conhecimento das repercussões imediatas e de longo prazo de uma alimentação inadequada tem contribuído para a busca de melhor entendimento de como o hábito alimentar é formado e chamado à atenção para a importância das práticas alimentares nos primeiros anos de vida (HART CN et al., 2010; BENTLEY ME e THOMPSON AL, 2013).

Segundo Adamo KB e Brett KE (2014), a qualidade da dieta infantil pode ser influenciada por fatores biológicos, as preferências e as práticas alimentares familiares, a disponibilidade de alimentos e a percepção dos pais. Este último pode ser uma ferramenta crucial, sendo capaz de influenciar positiva ou negativamente na alimentação ofertada à criança. Camargo APPM et al. (2013), afirma que os pais, especialmente a mãe, são elementos fundamentais em todo processo de alimentação e nutrição da criança, principalmente, em relação à ingestão de alimentos não saudáveis.

De acordo com Conte FA (2016), muitos alimentos importantes como frutas e vegetais in natura, cereais integrais, sementes, e tantos outros foram aos poucos sendo substituídos por produtos prontos que possuem praticidade, sabor mais acentuado e por uma infinidade de motivos que inclui desde as mudanças culturais à questão econômica. Segundo Bielemann RM et al. (2015), estes alimentos processados estão se tornando cada vez mais acessíveis para todas as faixas etárias. Polonio MLT e Peres F (2009) afirmam que é preciso ter um olhar amplo para a saúde e nutrição infantil, uma vez que diversos alimentos industrializados, que compõe cotidianamente a alimentação das crianças apresentam aditivos alimentares, que podem provocar efeitos adversos à saúde.

Segundo Ferreira FS (2015), devido as suas variadas funções, os aditivos químicos são cada vez mais inseridos precocemente e de forma elevada na alimentação trazendo sérios problemas de saúde a curto e longo prazo principalmente para as crianças que são as maiores consumidoras desses produtos, visto que, a indústria investe maciçamente na adição desses ingredientes nos alimentos para melhorar as suas características sensoriais, tornando-os mais atrativos e desejados. Pereira LF et al. (2015) afirmam que diversos estudos têm comprovado que tais aditivos podem apresentar toxicidade se não forem utilizados dentro de seus limites de segurança, podendo oferecer riscos, em especial, aos alérgicos a essas substâncias. 
De acordo com Polônio MLT (2010), a vulnerabilidade da população infantil aos efeitos dos aditivos químicos pode ser fundamentada pelo fato de a quantidade ingerida ser, em relação ao peso corporal, nomeadamente maior na criança, por que essas substâncias podem ser metabolizadas e excretadas de forma ineficaz devido à imaturidade fisiológica e por que as mesmas não apresentam capacidade de autocontrole no consumo de alimentos ricos em aditivos. Sendo assim, diante desta problemática, este trabalho teve como objetivo identificar os aditivos químicos mais prevalentes nos alimentos ultraprocessados consumidos pelas crianças e verificar os riscos que estes componentes podem trazer a saúde infantil.

\section{METODOLOGIA}

Trata-se de um estudo de delineamento transversal, com caráter descritivo qualitativo e quantitativo, dividido em duas partes, sendo a primeira a análise dos rótulos de produtos alimentícios ultraprocessados voltados ao público infantil quanto a presença de aditivos químicos, e a segunda, a relação dos riscos que os aditivos mais presentes podem trazer a saúde infantil. Inicialmente, para a delimitação da amostra realizouse uma pesquisa em 5 supermercados da cidade de Teresina-Piauí com o objetivo de conhecer os produtos ofertados.

Para a escolha dos alimentos adotou-se como critérios de inclusão: Produtos alimentícios que atendiam a definição e exemplificação de "ultraprocessados" segundo o Guia Alimentar para a População Brasileira 2014, que apresentavam em sua rotulagem e/ou marketing comercial, aspectos que caracterizam publicidade abusiva à criança conforme a RDC no 163/2014 do Conselho Nacional dos Direitos da Criança e do Adolescente - CONANDA e que continham simultaneamente as três marcas escolhidas nos cinco supermercados. A partir disso foram selecionadas 3 marcas de 17 alimentos ultraprocessados disponíveis nas prateleiras, totalizando 51 rótulos a serem analisados.

Para a coleta de dados realizou-se busca dos rótulos nos endereços eletrônicos (sites) das marcas selecionadas. Os dados foram organizados, de forma que fosse possível avaliar quantitativamente e qualitativamente a presença de aditivos. Os 17 tipos de alimentos ultraprocessados foram separados em 3 grupos descritos no quadro a seguir, sendo especificados como grupo I, grupo II e grupo III respectivamente.

Quadro 1 - Grupos de alimentos e produtos alimentícios analisados.

\begin{tabular}{|l|l|}
\hline \multicolumn{1}{|c|}{ GRUPOS DE ALIMENTOS } & \multicolumn{1}{c|}{ PRODUTOS ALIMENTícIOS } \\
\hline Doces & $\begin{array}{l}\text { Biscoitos recheado, biscoito wafer recheado, biscoito cookies, bolo } \\
\text { pronto, cereal matinal, gelatina, bala de goma, goma de mascar, pirulito } \\
\text { e confeitos de chocolate. }\end{array}$ \\
\hline Salgados & Salgadinho de milho e batata frita ondulada. \\
\hline Bebidas industrializadas & Achocolatado, leite fermentado, iogurte, suco pronto e refrigerante. \\
\hline
\end{tabular}

Fonte: Dados da pesquisa, 2018.

A relação das classes e tipos de aditivos químicos mais prevalentes nestes alimentos com os possíveis riscos à saúde foi realizada através de pesquisa bibliográfica nas bases de dados Scielo, PubMed e LILACS, utilizando-se os descritores: "alimentação infantil", "aditivos alimentares" e como critérios de inclusão os artigos datados de 2008 a 2018, que tratavam sobre as classes e os tipos de aditivos de interesse e os que descreviam quais riscos que os mesmos podem trazer a saúde infantil. Dessa forma, utilizou-se no estudo 20 artigos científicos. Para a análise estatística dos resultados, utilizou-se o Programa Microsoft Excel® para organização e análise dos dados e as variáveis foram apresentadas por meio de estatística descritiva: números e proporções. 


\section{RESULTADOS E DISCUSSÃO}

Foram analisados 51 rótulos de produtos alimentícios destinados ao público infantil. No gráfico 1, estão descritas as classes de aditivos encontradas em maior frequência nestes alimentos, sendo elas os aromatizantes, presentes em $98 \%$ dos rótulos, corantes em $67 \%$ e acidulantes em $57 \%$.

Gráfico 1 - Classes de aditivos mais prevalentes nos alimentos ultraprocessados.

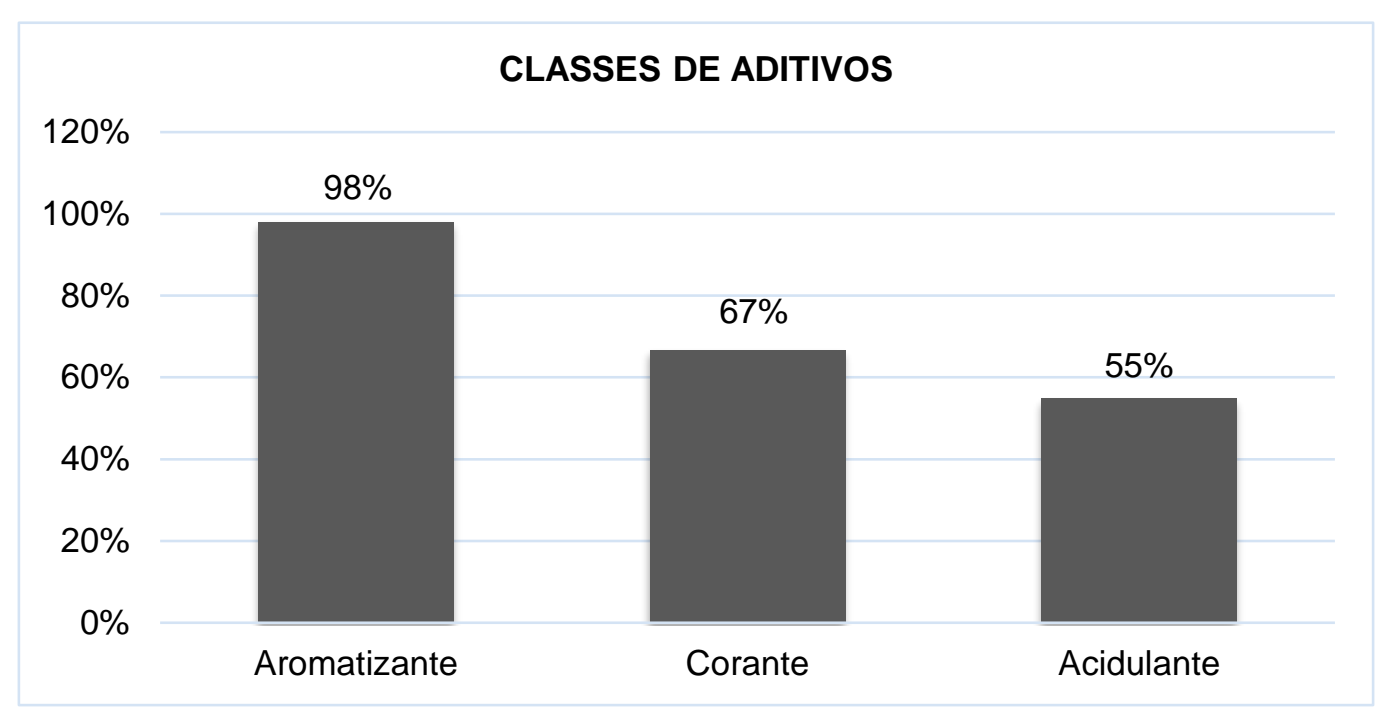

Fonte: Dados da pesquisa, 2018.

Em um estudo realizado por Pereira LF et al. (2015), os resultados indicaram que a classe dos aromatizantes também esteve entre as mais prevalentes em todas as categorias de alimentos avaliadas. No trabalho realizado por Silva JB (2016) que analisou produtos alimentícios ofertados à população infantil, e no trabalho de Albuquerque MV (2012) também se observou este mesmo resultado. Porém, em relação aos efeitos que este aditivo pode gerar a saúde infantil há poucos estudos disponíveis no meio cientifico. Segundo Honorato TC et al. (2013), eles possuem especial importância por conferirem propriedades sensórias que caracterizam cada sabor e aroma dos mais diversos produtos, e grande parte do sabor de um alimento é diretamente influenciado pelo seu aroma já que em meio a uma grande variedade de opções, são as características diferenciais que vão determinar a aceitação do produto pelo consumidor.

De acordo com a Resolução - RDC no 2, de 15 de Janeiro de 2007, os aromatizantes podem ser classificados como naturais e sintéticos, este último compreende os idênticos ao natural e os artificiais. Lerner CA et al. (2015) relatam que o uso destes aditivos gera perguntas sobre sua toxicidade em nível sistêmico e celular, e há uma necessidade urgente de realizar pesquisas para avaliar seu potencial toxicológico. Moura AG et al. (2016) também afirmam que peritos da área de segurança alimentar declaram que os aditivos de aroma e sabor, com ênfase aos sintéticos, suscitam uma série de dúvidas quanto aos seus potenciais efeitos citotóxicos, genotóxicos e mutagênicos, uma vez que, estudos toxigenéticos de tais substâncias são praticamente inexistentes na literatura científica.

Ainda de acordo com Honorato TC et al. (2013), não há perigo de toxicidade nos classificados como naturais, já nos artificiais, quando aplicados em baixa dose, não há risco, porém, quando as doses são elevadas, podem provocar ações irritantes e narcóticas e outros produzir toxicidade crônica em longo prazo. A literatura destaca também que doses elevadas de aromatizantes sintéticos podendo trazer problemas de retardo de crescimento infantil e câncer quando há uma exposição excessiva e prolongada dessas substâncias (FERREIRA FS, 2015). Brown G (2008), afirma que os aromatizantes sintéticos podem provocar ações irritantes na região do estômago, porém não foi observado indicativo de toxicidade sistêmica direta. 
No presente estudo $78 \%$ dos rótulos que continham esta classe de aditvo não especificaram qual o tipo estava sendo utilizado, o que impossibilitou sua classificação, diferentemente dos corantes e acidulantes (Gráfico 2 e 3). Pereira LF et al. (2015) também relata na sua pesquisa que nos rótulos dos alimentos não foram detalhados a origem dos aromatizantes adicionados, podendo estes serem sintéticos ou naturais e isto acaba gerando preocupações em relação aos efeitos que pode causar a saúde infantil visto que não é possível ter conhecimento sobre qual tipo está sendo ingerido, além disso, nos mostra uma lacuna na legislação, pois a mesma não exige que as empresas determinem na rotulagem qual tipo está sendo utilizado no aliemento.

Diferente dos aromatizantes, segundo Al- Shabib NA et al. (2017), nos ultimos anos os corantes vêm sendo largamente estudados devido à possível toxicidade, visto que existem relatos de correlação com reações alérgicas, hiperatividade em crianças, genotoxicidade, tumor da tireóide, Transtorno do Déficit de Atenção e Hiperatividade - TDHA e urticária. Anastácio LB et al. (2016), afirma que a toxicidade dos artificiais e os riscos que estes podem causar é objeto de discussão atualmente, visto que, alergias, rinite, broncoconstrição, hiperatividade, danificação cromossômica ou tumores, têm sido reportados pela literatura. De acordo com Siqueira APC et al. (2011), quando são utilizados em níveis superiores aos permitidos pelas legislações podem ser tóxicos e as consequências vão desde alergias até riscos de câncer.

De acordo com Sá P et al. (2016), em 2013 no Brasil, foi realizado um estudo exploratório-descritivo sobre o uso de corantes artificiais e observou-se que eles estão diretamente relacionados com a hiperatividade e em 2015, nos Estados Unidos, outro estudo concluiu que o consumo pode conduzir a reações cruzadas, autoimunes e até mesmo transtornos neurocomportamentais. Estudos recentes também mostram que os artificiais são cancerígenos, podem causar dermatite alérgica e irritação da pele (HAMERSKI L, et al., 2013). Polônio MLT e Peres F (2009), afirmam que essas substâncias podem ocasionar o aparecimento de TDHA e alguns estudos demonstraram melhora no quadro clínico da hiperatividade em crianças submetidas a uma dieta isenta desses compostos químicos.

Feketea G e Tsabouri S (2017) verificaram o surgimento de alergias em crianças e concluíram que, os corantes artificiais podem agravar dermatite atópica existente, causar sintomas de dermatite, urticaria, angioedema, asma, choque anafilático e eczema. Arnold L et al. (2012), ao realizarem um estudo por meio de uma revisão bibliográfica, mostraram que o consumo de produtos com esse tipo de corante esteve relacionado com relatos de hiperatividade e déficit de atenção em crianças, tanto por determinação médica quanto por opinião parental. De acordo com Pinheiro COM e Abrantes SMP (2012), é importante ressaltar que a legislação brasileira não obriga os fabricantes de alimentos a especificarem a quantidade deste aditivo empregada em seus produtos, sendo apenas necessário especificar quais os que o alimento contém. Dessa forma, há uma facilidade de o público infantil ultrapassar a Ingestão Diária Aceitável - IDA recomendada visto que não consomem apenas um produto contendo este aditivo. Nos gráficos 2 e 3, estão descritos os tipos de corantes e de acidulantes encontrados em maior frequência nos produtos ultraprocessados analisados.

Nesta pesquisa, os corantes artificiais foram os mais frequentes, sendo o Azul Brilhante o mais prevalente, estando em $47 \%$ dos rótulos analisados, seguido do Vermelho 40 em 35\% e Tartrazina em $32 \%$ (Gráfico 2). Citação retirada.

Assim como nesta pesquisa, Pinheiro COM e Abrantes SMP (2012) também verificaram que dentre os corantes que mais constavam nos rótulos das balas consumidas pelas crianças estavam o Azul brilhante e 0 Vermelho 40. De acordo com Barros AA e Barros EBP (2010), o Azul Brilhante pertence ao grupo dos trifenilmetanos e seu uso foi banido nos Estados Unidos, mas autorizado na Europa, fazendo parte de um grupo onde muito se discute quanto a sua real segurança, por isso seu uso é controlado em certos países. Segundo Polônio MLT e Peres F (2009), seu consumo pode causar hiperatividade em crianças, eczema e asma.

De acordo com Freitas AS (2012), dentre os aditivos mais genotóxicos existentes estão os pertencentes ao grupo "Azo", um derivado nitroso capaz de ocasionar reações de hipersensibilidade e tem sido foco de estudos de mutagênese e carcinogênese por produzir, após ser metabolizado pela microflora intestinal, compostos com altos potenciais cancerígenos, além disso, outro fator negativo causado pelo consumo de 
corantes azo é o desencadeamento de processos alérgicos. Segundo Gomes LMM (2012), o Vermelho 40 e a Tartrazina estão entre os que fazem parte desse grupo e são encontrados principalmente em sucos artificiais, balas, gelatinas, iogurtes e refrigerantes.

Gráfico 2 - Tipos de corantes mais prevalentes nos alimentos ultraprocessados.

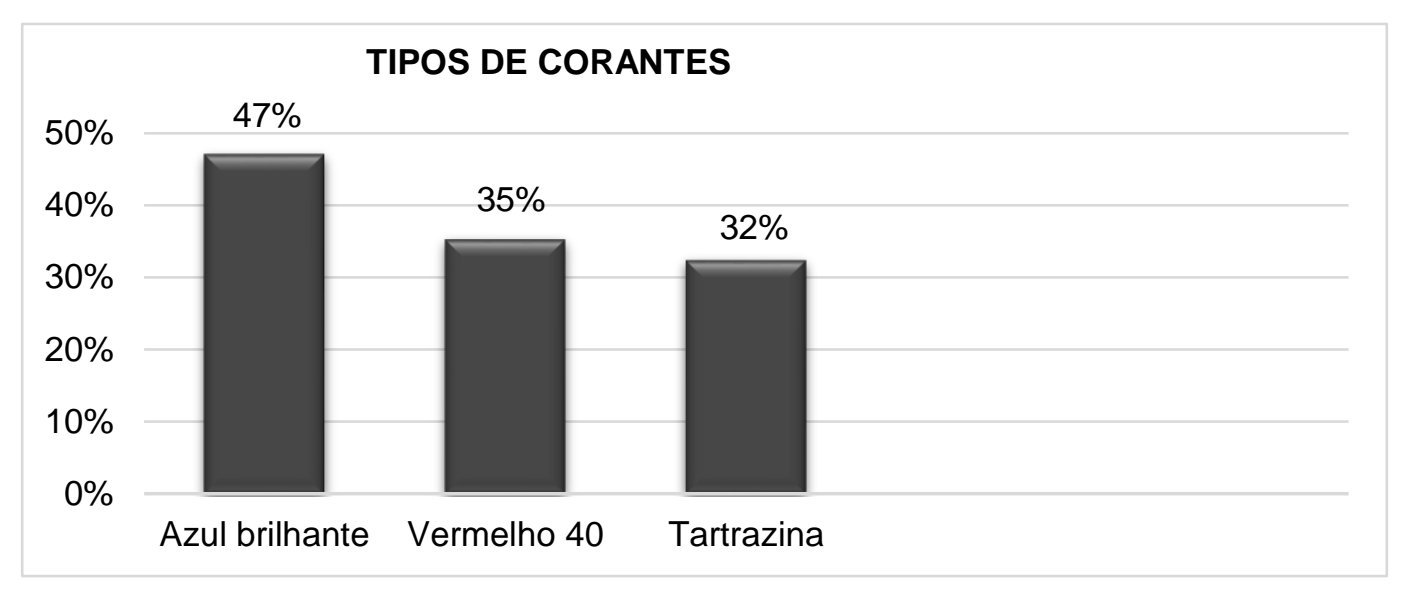

Fonte: Dados da pesquisa, 2018.

Em uma pesquisa realizada por Valente $\mathrm{MCH}$ (2018), quando analisadas as dietas das crianças individualmente, dentre os corantes apresentados nos alimentos relatados, o Vermelho 40 teve sua IDA ultrapassada por algumas crianças. Segundo Feketea G e Tsabouri S (2017), em relação à reação alérgica nas crianças, não foi reportado nenhum caso mediado por ele. Entretanto, de acordo com Leo L et al. (2017), um estudo efetuado com neutrófilos humanos notou que 0 aditivo aumenta a produção de mediadores inflamatórios através dessas células. Já Câmara AM (2017), afirma que o Vermelho 40 tem sido relatado como possível influenciador nos níveis de hiperatividade em crianças.

No trabalho de McCann D et al. (2007), que teve como objetivo observar o impacto do consumo de corantes artificiais sobre o comportamento de crianças, utilizando bebidas feitas com misturas de vários corantes, majoritariamente da classe azo, foi possível concluir que, as crianças que consumiram os mesmos apresentaram-se mais hiperativas e menos atentas. De acordo com Freitas AS (2012), dentre os do tipo "azo", a tartrazina tem o maior respaldo sendo relacionada com diversas reações de hipersensibilidade como urticária, asma, náusea, anafilaxia, vômitos, dermatite, dor de cabeça, eczema e em doses elevadas induz à lesão no DNA possibilitando o surgimento de câncer em longo prazo.

A Tartrazina é um corante azoico utilizado para conferir a cor amarela a vários alimentos, tais como sorvetes, bolos, balas e confeitos, salgadinhos de batata, refrigerantes, bebidas alcóolicas, chicletes, gelatina, entre outros (AL-SHABIB NA, et al., 2017). Uma pesquisa publicada por Stevenson J et al. (2007), mostrou que misturas de aditivos, comumente achadas em alimentos, que continham Tartrazina e Vermelho 40, quando administrada em alimentos infantis, causava aumento da hiperatividade nas crianças de 3 a 9 anos de idade e demonstraram que o uso destes aditivos acentua comportamentos como desatenção e impulsividade. Segundo Sá P et al. (2016), dentre os aditivos relacionados ao Transtorno de Déficit de Atenção e Hiperatividade em crianças, a Tartrazina foi o mais implicado.

De acordo com Matsuo $\mathrm{H}$ et al. (2013), a maioria das crianças inicia o consumo de produtos com a presença de Tartrazina antes dos 2 anos de idade, e o pó para gelatina era introduzido até 1 ano em 95\% dos casos. No estudo realizado por Ward NI (1997), foi possível observar que 23 crianças que consumiram bebidas contendo tartrazina, destas, 18 aumentaram os níveis de hiperatividade, 16 se tornaram agressivas, 4 violentas, 2 diminuíram seus movimentos, 12 tiveram diminuição da coordenação motora e 8 desenvolveram asma. 
Gráfico 3 - Tipos de acidulantes mais prevalentes nos alimentos ultraprocessados.

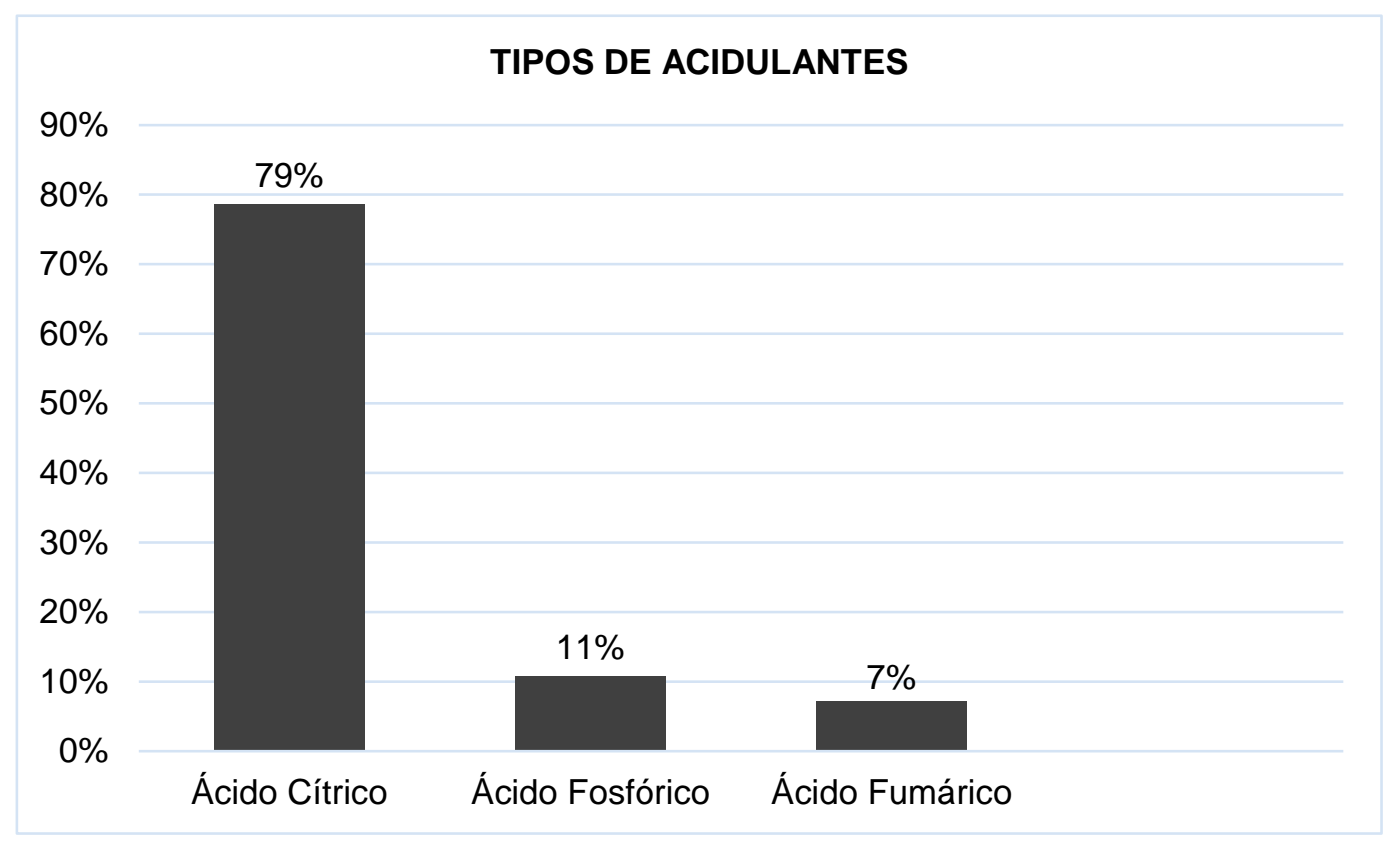

Fonte: Dados da pesquisa, 2018.

Em relação a classe dos acidulantes, o ácido cítrico (82\%), ácido fosfórico (11\%) e ácido fumárico (7\%) foram os encontrados em maior prevalência nos alimentos. (Gráfico 3).

De acordo com Silva MV et al. (2009) e Pires DAT e Machado PFL (2013), o ácido cítrico, um dos acidulantes mais comumente usados na indústria de alimentos, pode agir como redutor do $\mathrm{pH}$. Alguns sintomas provocados pela administração de grandes doses aumentam a atividade geral, aparecendo hiperpnéia, vaso dilatação periférica, salivação, convulsões crônicas e tônicas.

Segundo Oliveira ACS et al. (2011), o ácido fosfórico, é um ácido que tem por função conservar o alimento, assim como intensificar o sabor. Porém esse ácido pode trazer malefícios às crianças, visto que quando ingerido há diminuição do $\mathrm{pH}$ do corpo, e este precisa estar em equilíbrio, para isso o corpo retira o cálcio dos ossos, o que pode levar a uma osteoporose no futuro, prejuízo no crescimento e desenvolvimento. Polônio MLT e Peres F (2009) e Ferreira FS (2015), também afirmam que devido ao excesso de acidulantes, como o ácido fosfórico, pode haver principalmente nas crianças, descalcificação dos dentes e dos ossos, levando ao enfraquecimento.

\section{CONCLUSÃO}

As classes dos aromatizantes, corantes e acidulantes, encontradas em maior prevalência nos produtos alimentícios analisados estão relacionadas a diversos prejuízos a saúde infantil. Entre estes problemas podem-se destacar: alergias, com a presença de urticária, angioedema, broncoespasmo e choque, Transtorno do Déficit de Atenção e Hiperatividade, retardo do crescimento infantil, vários tipos de câncer, descalcificação dos dentes e dos ossos, que levam ao enfraquecimento, entre muitos outros. Porém, apesar destes relatos, nota-se que em relação a alguns aditivos as pesquisas abordando os efeitos que o consumo dos mesmos pode ocasionar a saúde das crianças ainda são escassas. Sendo assim, fica evidente a necessidade de mais investimentos em pesquisas nesta área e torna-se imprescindível que haja um incentivo para redução no consumo de alimentos que contém estas substâncias, tendo em vista as evidências já mencionadas no meio científico. 


\section{REFERÊNCIAS}

1. ADAMO KB, BRETT KE. Parental perceptions and childhood dietary quality. Matern. Child Health J, 2014; 18(4): 978-95.

2. ALBUQUERQUE MV. Educação alimentar: Uma proposta de redução do consumo de aditivos alimentares. Química Nova na Escola, 2012; 34(2): 51-57.

3. AL-SHABIB NA, et al. Synthetic food additive dye Tartrazine triggers amorphous aggregation in cationic myoglobin. International Journal of Biological Macromolecules, 2017; 98(1):277-286.

4. ANASTÁCIO LB, et al. Corantes alimentícios amaranto, eritrosina B e tartrazina, e seus possíveis efeitos maléficos à saúde humana. Journal of Applied Pharmaceutical Sciences - JAPHAC, 2016; 2(3):16-30.

5. ARNOLD L, et al. Artificial food colors and attention-deficit hyperactivity symptoms: conclusions to dye for. Neurotherapeutics, 2012; 9(3): 599-609.

6. BARROS AA, BARROS EBP. A química dos alimentos: produtos fermentados e corantes. Sociedade Brasileira de Química. São Paulo: Edit-SBQ, 2010; 88P.

7. BENTLEY ME, THOMPSON AL. The critical period of infant feedingfor the development of early disparities in obesity. Soc Sci Med, 2013; 97(1): 288-96.

8. BIELEMANN RM, et al. Consumo de alimentos ultraprocessados e impacto na dieta de adultos jovens. Revista de Saúde Pública, 2015; 49(28): 1-10.

9. BRASIL. Agência Nacional de Vigilância Sanitária. Resolução - RDC № 2, de 15 de Janeiro de 2007. Regulamento Técnico sobre Aditivos Aromatizantes. Brasília (DF): Anvisa; 2007.

10. BROWN G. Review of the white blood cell data on a series of toxicity studies for Huntingdon Life Sciences. Laboratorios Miret S.A, 2008.

11. CÂMARA AM. Corantes azo: características gerais, aplicações e toxicidade. Dissertação (Graduação em Nutrição) - Centro de Ciências da Saúde. Departamento de Nutrição. Universidade Federal do Rio Grande do Norte, Natal, 2017; 60p.

12. CAMARGO APPM, et al. A não percepção da obesidade pode ser um obstáculo no papel das mães de cuidar de seus filhos. Cienc. Saúde Colet, 2013; 18 (2): 323-33.

13. CONTE FA. Efeitos do consumo de aditivos químicos alimentares na saúde humana. Revista Espaço Acadêmico, 2016; 16 (181): 69-81.

14. FEKETEA G, TSABOURI S. Common food colorants and allergic reactions in children: Myth or reality. Food Chemistry, 2017; 230(1): 578588.

15. FERREIRA FS. Aditivos alimentares e suas reações adversas no consumo infantil. Rev da Universidade Vale do Rio Verde, 2015; 13(1): 397-407.

16. FREITAS AS. Tartrazina: uma revisão das propriedades e análises de quantificação. Acta Tecnológica, 2012; $7(2): 65-72$.

17. GOMES LMM. Inclusão de carotenóides de pimentão vermelho em ciclodextrinas e avaliação da sua estabilidade, visando aplicação em alimentos. Dissertação (Mestre em Ciências Aplicadas) - Faculdade de Farmácia. Universidade Federal Fluminense, Niterói, 2012; 108 p.

18. HAMERSKI L, et al. Usando as cores da natureza para atender aos desejos do consumidor: Substâncias naturais como corantes na indústria alimentícia. Rev Virtual Quim, 2013; 5(3): 394-420.

19. HART CN, et al. The association of maternal food intake and infants and toddlers food intake. Child Care Health Dev, 2010; 37(3): 396-403.

20. HONORATO TC, et al. Aditivos alimentares: aplicações e toxicologia. Revista Verde de Agroecologia e Desenvolvimento Sustentável, 2013; 18(5): 1-11.

21. LEO L, et al. Occurrence of Azo Food Dyes and their Effects on Cellular Inflammatory Responses. Nutrition, 2017; 46 (1): 36-40.

22. LERNER CA, et al. Vapors produced by electronic cigarettes and e-juices with flavorings induce toxicity, oxidative stress, and inflammatory response in lung epithelial cells and in mouse lung. PLoS One, 2015; 10(2): 2-10.

23. MATSUO H, et al. Aspirin Augments lgE-mediated histamine release from human peripheral basophils via syk kinase activation. Allergology International, 2013; 62(4): 503-511.

24. MCCANN D, et al. Food additives and hyperactive behavior in 3-year-old and 8/9-year-old children in the community: A randomized, double-blinded, placebocontrolled. Lancet, 2007; 370(1): 560-1567.

25. MOURA AG, et al. Cytotoxicity of cheese and cheddar cheese food flavorings on Allim cepa $L$ root meristems. Brazilian Journal of Biology, 2016; 76(2): 439-443.

26. OLIVEIRA ACS, et al. Impacto do consumo de refrigerantes na saúde de escolares do colégio Gissoni. Revista Eletrônica Novo Enfoque, 2011; 12(12): 68-79.

27. PEREIRA LF, et al. Prevalência de Aditivos em Alimentos Industrializados Comercializados em uma Cidade do Sul de Minas Gerais. Rev Ciências em Saúde, 2015; 5(3): 1-7. 
28. PINHEIRO COM, ABRANTES SMP. Determinação dos corantes artificiais presentes em balas consumidas por crianças com idade entre 3 e 9 anos. Revista Analytica, 2015; 13(1): 10-23.

29. PIRES DAT, MACHADO PFL. Refrigerante e bala de menta: Explorando possibilidades. Química nova na escola, 2013; 35(3): 166-173.

30. POLÔNIO MLT. Percepção de mães quanto aos riscos à saúde de seus filhos em relação ao consumo de aditivos alimentares: o caso dos pré-escolares do município de Mesquita. Tese (Doutorado em Ciências na área de Saúde Pública e Meio Ambiente) - Escola Nacional de Saúde Pública Sergio Arouca, Rio de Janeiro, 2010; 129 p.

31. POLÔNIO MLT, PERES F. Consumo de aditivos alimentares e efeitos à saúde: desafios para a saúde pública brasileira. Cad. Saúde Pública, 2009; 25(8): 1653-1666.

32. SÁ P, et al. Uso abusivo de aditivos alimentares e transtornos de comportamento: há uma relação?. International Journal of Nutrology, 2016; 9(2): 209-215.

33. GONÇALVES ECBA, et al. Avaliação do consumo de corantes artificiais por lactentes, pré-escolares e escolares. Ciência e Tecnologia de Alimentos. 2008; 28(3): 534-39.

34. SILVA JB. Análise de produtos alimentícios ofertados à população infantil: tipo de processamento e presença de aditivos químicos. Dissertação (Graduação em Nutrição) - Universidade Federal do Rio Grande do Norte, Natal, 2016; 97 p.

35. SILVA MV, et al. Conceitos e métodos de controle do escurecimento enzimático no processamento mínimo de frutas e hortaliças. Centro de pesquisa e processamento de alimentos, 2009; 27(1): 83-96.

36. SIQUEIRA APC, et al. Desenvolvimento de métodos analíticos para determinação corantes em amostras de sucos e gelatinas. Revista eletronica interdisciplinar, 2011; 1(17): 263-269.

37. STEVENSON J, et al. Food additives and hyperactive behaviour in 3-yaer-old and 8/9-year-old children in the community: a randomized, double- -blinded, placebo-controlled trial. The Lancet, 2007; 370(9598): 1560-1567.

38. VALENTE MCH. Corantes artificiais: Estudo da estimativa de ingestão por crianças e da percepção de adultos residentes no rio grande do sul. Dissertação (Pós-Graduação em Ciência e Tecnologia de Alimentos) Universidade Federal do Rio Grande do Sul, Porto Alegre, 2018; 139 p.

39. WARD NI. Assessment of chemical factors in relation to child hyperactivity. J. Nutr. Environ, 1997; 7(4): 333-3.

40. POLÔNIO MLT, PERES F. Consumo de aditivos alimentares e efeitos à saúde: desafios para a saúde pública brasileira. Cad. Saúde Pública, 2009; 25(8): 1653-1666.

41. SÁ P, et al. Uso abusivo de aditivos alimentares e transtornos de comportamento: há uma relação?. International Journal of Nutrology, 2016; 9(2): 209-215.

42. GONÇALVES ECBA, et al. Avaliação do consumo de corantes artificiais por lactentes, pré-escolares e escolares. Ciência e Tecnologia de Alimentos. 2008; 28(3): 534-39.

43. SILVA JB. Análise de produtos alimentícios ofertados à população infantil: tipo de processamento e presença de aditivos químicos. Dissertação (Graduação em Nutrição) - Universidade Federal do Rio Grande do Norte, Natal, 2016; 97 p.

44. SILVA MV, et al. Conceitos e métodos de controle do escurecimento enzimático no processamento mínimo de frutas e hortaliças. Centro de pesquisa e processamento de alimentos, 2009; 27(1): 83-96.

45. SIQUEIRA APC, et al. Desenvolvimento de métodos analíticos para determinação corantes em amostras de sucos e gelatinas. Revista eletronica interdisciplinar, 2011; 1(17): 263-269.

46. STEVENSON J, et al. Food additives and hyperactive behaviour in 3-yaer-old and 8/9-year-old children in the community: a randomized, double- -blinded, placebo-controlled trial. The Lancet, 2007; 370(9598): 1560-1567.

47. VALENTE MCH. Corantes artificiais: Estudo da estimativa de ingestão por crianças e da percepção de adultos residentes no rio grande do sul. Dissertação (Pós-Graduação em Ciência e Tecnologia de Alimentos) Universidade Federal do Rio Grande do Sul, Porto Alegre, 2018; 139 p.

48. WARD NI. Assessment of chemical factors in relation to child hyperactivity. J. Nutr. Environ, 1997; 7(4): 333-3. 